\title{
Percutaneous placement of lumbar pedicle screws via intraoperative CT image-based augmented reality-guided technology
}

\author{
Huan Liu, MD, Junlong Wu, MD, Yu Tang, MD, PhD, Haiyin Li, MD, PhD, Wenkai Wang, MMed, \\ Changqing Li, MD, PhD, and Yue Zhou, MD, PhD
}

Department of Orthopedics, The Second Affiliated Xinqiao Hospital of Army Medical University, Chongqing, China

OBJECTIVE The authors aimed to assess, in a bone-agar experimental setting, the feasibility and accuracy of percutaneous lumbar pedicle screw placements using an intraoperative CT image-based augmented reality (AR)-guided method compared to placements using a radiograph-guided method. They also compared two AR hologram alignment methods.

\begin{abstract}
METHODS Twelve lumbar spine sawbones were completely embedded in hardened opaque agar, and a cubic marker was fixed on each phantom. After intraoperative CT, a 3D model of each phantom was generated, and a specialized application was deployed into an AR headset (Microsoft HoloLens). One hundred twenty pedicle screws, simulated by Kirschner wires (K-wires), were placed by two experienced surgeons, who each placed a total of 60 screws: 20 placed with a radiograph-guided technique, 20 with an AR technique in which the hologram was manually aligned, and 20 with an AR technique in which the hologram was automatically aligned. For each $\mathrm{K}$-wire, the insertion path was expanded to a $6.5-\mathrm{mm}$ diameter to simulate a lumbar pedicle screw. CT imaging of each phantom was performed after all K-wire placements, and the operative time required for each K-wire placement was recorded. An independent radiologist rated all images of K-wire placements. Outcomes were classified as grade I (no pedicle perforation), grade II (screw perforation of the cortex by up to $2 \mathrm{~mm}$ ), or grade III (screw perforation of the cortex by $>2 \mathrm{~mm}$ ). In a clinical situation, placements scored as grade I or II would be acceptable and safe for patients.
\end{abstract}

RESULTS Among all screw placements, $75(94 \%)$ of 80 AR-guided placements and $40(100 \%)$ of 40 radiograph-guided placements were acceptable (i.e., grade I or II; $p=0.106$ ). Radiograph-guided placements had more grade I outcomes than the AR-guided method $(p<0.0001)$. The accuracy of the two AR alignment methods $(p=0.526)$ was not statistically significantly different, and neither was it different between the AR and radiograph groups $(p<0.0001)$. AR-guided placements required less time than the radiograph-guided placements (mean \pm standard deviation, $131.76 \pm 24.57$ vs 181.43 \pm 15.82 seconds, $p<0.0001)$. Placements performed using the automatic-alignment method required less time than those using the manual-alignment method ( $124.20 \pm 23.80$ vs $139.33 \pm 23.21$ seconds, $p=0.0081$ ).

CONCLUSIONS In bone-agar experimental settings, AR-guided percutaneous lumbar pedicle screw placements were acceptable and more efficient than radiograph-guided placements. In a comparison of the two AR-guided placements, the automatic-alignment method was as accurate as the manual method but more efficient. Because of some limitations, the AR-guided system cannot be recommended in a clinical setting until there is significant improvement of this technology.

https://thejns.org/doi/abs/10.3171/2019.10.SPINE19969

KEYWORDS lumbar pedicle screw; augmented reality; spinal surgery; Kirshner wire; surgical technique

A UGMENTED reality (AR) is an interactive experience simulating a real-world environment wherein the objects are "augmented" by computer-generated perceptual information. With the help of advanced AR technologies, such as computer vision and object recogni- tion, information on the surrounding real world becomes interactive and manipulatable by the user. Information on the environment and its objects is overlaid onto the real world. ${ }^{2,4,11}$ This technology makes it possible to superimpose real-time images or 3D structures into the user's view,

ABBREVIATIONS AR = augmented reality; K-wire = Kirschner wire; MIS = minimally invasive spine; STL = Standard Tessellation Language.

SUBMITTED August 19, 2019. ACCEPTED October 8, 2019.

INCLUDE WHEN CITING Published online December 20, 2019; DOI: 10.3171/2019.10.SPINE19969. 
which could be helpful in directing imaging-guided interventions. ${ }^{7,8}$ The Microsoft HoloLens (Microsoft Corp.) is an AR headset with an integrated holographic processing unit that allows 3D holograms to be spatially projected into the wearer's view.

Recently, minimally invasive spine (MIS) procedures have become widespread in spinal surgery. ${ }^{17,20}$ An MIS procedure is "one that by virtue of the extent and means of surgical technique results in less collateral tissue damage, resulting in [a] measurable decrease in morbidity and more rapid functional recovery than traditional exposures, without differentiation in the intended surgical goal."13 MIS procedures reduce blood loss, decrease postoperative back pain, and shorten the hospital length of stay compared to those with traditional open procedures. $5,6,15$ However, a significant learning curve is also involved in attaining proficiency with MIS procedures and instrumentations, and complication rates are higher during this learning period..$^{10,16,19}$ Percutaneous lumbar pedicle screw placement is a routine operation in MIS procedures, and the accuracy of screw placement is highly dependent on the guidance by intraoperative fluoroscopy. AR technology could provide an overlay of not only the intraoperative fluoroscopic images, but also the patient's 3D anatomical structures, which could improve the surgeon's experience and provide an easier way to overcome the learning curve with MIS procedures.

Here, we aimed to assess, in bone-agar experimental settings, the feasibility and accuracy of percutaneous lumbar pedicle screw placements using a CT image-based AR-guided method compared to placements using a radiograph-guided method. We also compared two AR holographic methods of alignment.

\section{Methods}

Twelve lumbar spine sawbones were completely embedded in hardened opaque agar, and a $4 \times 4 \times 2-\mathrm{cm}$ cubic marker was fixed on each phantom. A $4 \times 4-\mathrm{cm}$ adhesive sticker with a unique printed image was pasted on the square surface of each cubic marker (Fig. 1). For each phantom, 10 lumbar pedicle screws could be placed (bilateral L1-5). Lumbar pedicle screw placements on all phantoms were divided into the following two groups based on the imaging method: AR guided $(n=80)$ or radiograph guided $(\mathrm{n}=40)$. The AR-guided group was further divided into two subgroups: automatic alignment $(n=40)$ and manual alignment $(n=40)$.

A CT scan (LightSpeed VCT64, GE Healthcare) of each phantom was acquired at $120 \mathrm{kV}$ and $400 \mathrm{~mA}$ with a slice thickness of $0.625 \mathrm{~mm}$. Digital Imaging and Communications in Medicine (DICOM) data on each CT scan were exported and loaded into segmentation software (Mimics, Materialise) to create 3D models of the different parts of each phantom by using the Threshold function. All 3D models, including lumbar vertebrae, lumbar discs, and cubic markers, were exported to Standard Tessellation Language (STL) files.

For each phantom in the AR-guided group, the STL file was imported into an integrated development environment recommended by Microsoft technical documents. The AR

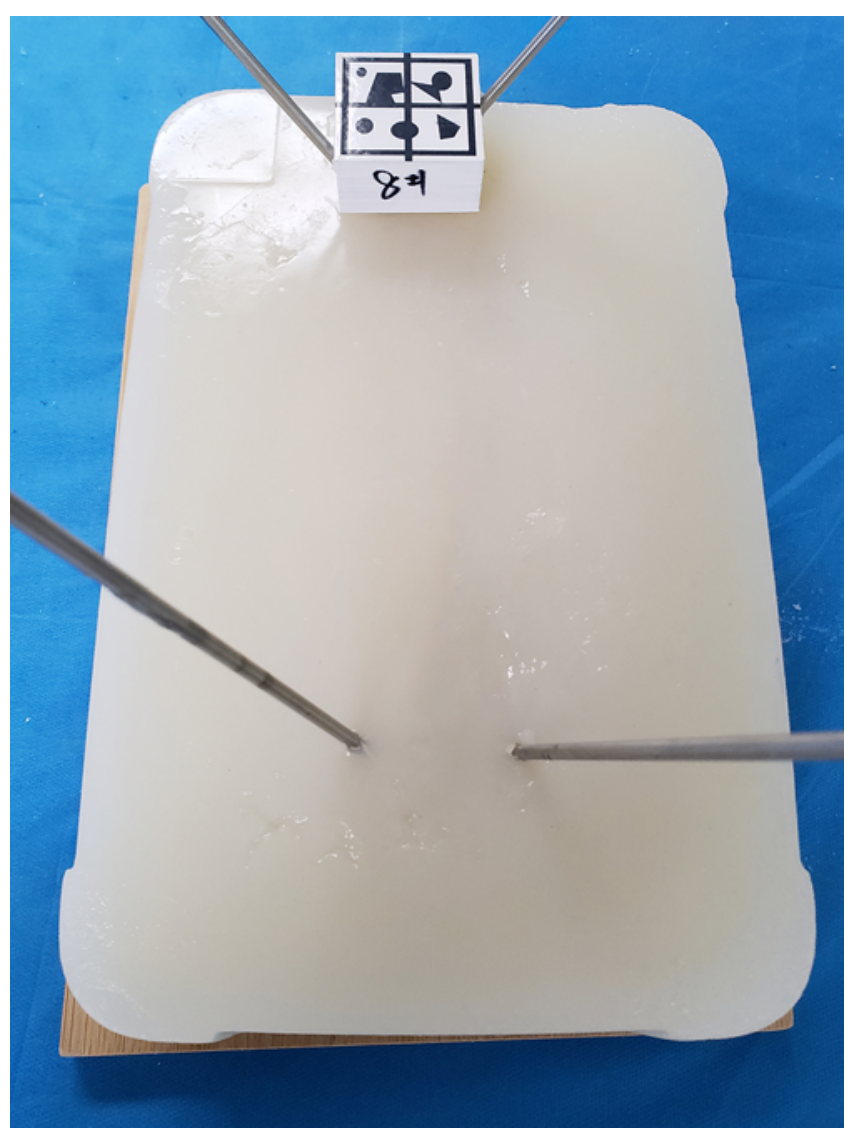

FIG. 1. A cubic marker was fixed on a spine phantom. Figure is available in color online only.

guiding application for each phantom was programmed and deployed to the Microsoft HoloLens. For the automatic-alignment subgroup, holograms were automatically aligned to the cubic marker using the image identification function. For the manual-alignment subgroup, the application provided the ability to move, resize, and rotate holograms to align to the cubic marker manually.

Two experienced spine surgeons independently performed 20 AR-guided percutaneous lumbar pedicle screw placements with automatic alignment, 20 AR-guided percutaneous lumbar pedicle screw placements with manual alignment, and 20 radiograph-guided percutaneous lumbar pedicle screw placements.

A total of 80 percutaneous lumbar pedicle screw placements were guided by AR technology. The spine surgeons used the guiding software for each phantom. Once the hologram was aligned automatically or manually, depending on the group, the surgeons targeted the bone surface using a percutaneous lumbar puncture needle (PAK Needle Pedicle Access Kit, Medtronic) and attempted to place a Kirschner wire (K-wire; $300 \mathrm{~mm}$ in length and $2.5 \mathrm{~mm}$ in diameter) into the pedicle at a $45-\mathrm{mm}$ depth limitation to simulate pedicle screw placement (Figs. 2 and 3). Both the outer width and outer height of each pedicle were measured to be larger than $7.8 \mathrm{~mm}$. To avoid perforation of the pedicle cortex, the pedicle screw diameter should be $1 \mathrm{~mm}$ less than the outer width or outer height of the pedicle.9,14 


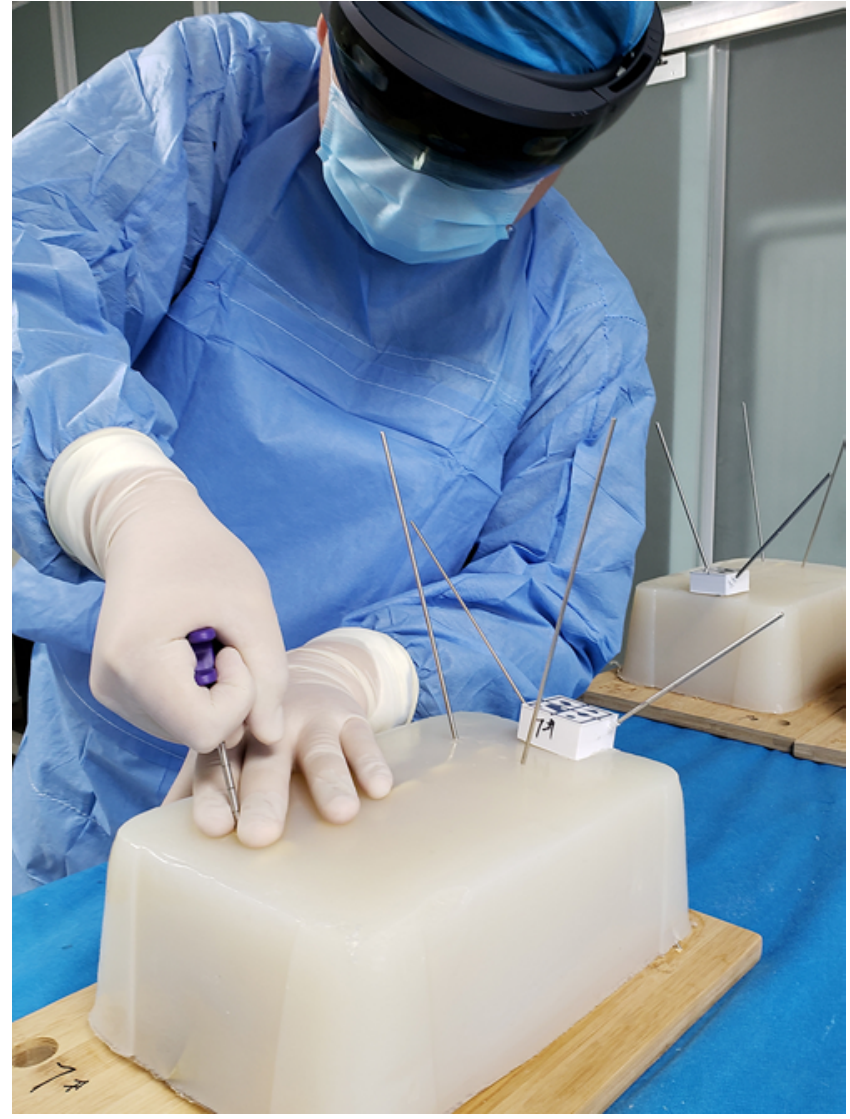

FIG. 2. Spine surgeons targeted the bone surface using a needle. Figure is available in color online only.

For each K-wire, the insertion path was expanded to a 6.5$\mathrm{mm}$ diameter to simulate a lumbar pedicle screw used in real surgical practice (Fig. 4). In total, 40 percutaneous lumbar pedicle screw placements were guided by radiography (G-arm, Whale Imaging). K-wires were placed into the pedicles in the same manner as is done in our clinical routine with the guidance of posteroanterior and lateral radiographic images.

CT scanning of each phantom was performed after all $\mathrm{K}$-wire placements. The operative time required for each placement from needle setup to finishing K-wire placement was recorded. An independent radiologist rated all images of $\mathrm{K}$-wire placements in a randomized order. The radiologist was blinded to the guiding method and experimental group. Outcomes were classified as grade I (no pedicle perforation), grade II (screw perforation of the cortex by up to $2 \mathrm{~mm}$ ), and grade III (screw perforation of the cortex by $>2 \mathrm{~mm}$ ). In a clinical situation, placements scored as grade I or II would be acceptable and safe for patients.

The accuracy of and operative time required for each placement were compared between the AR-guided and radiograph-guided groups. The two AR alignment subgroups were also compared. The accuracy of the guiding method or alignment method was analyzed using the chisquare test. The operative time for each placement among the guiding methods was analyzed with a two-sample Wil-

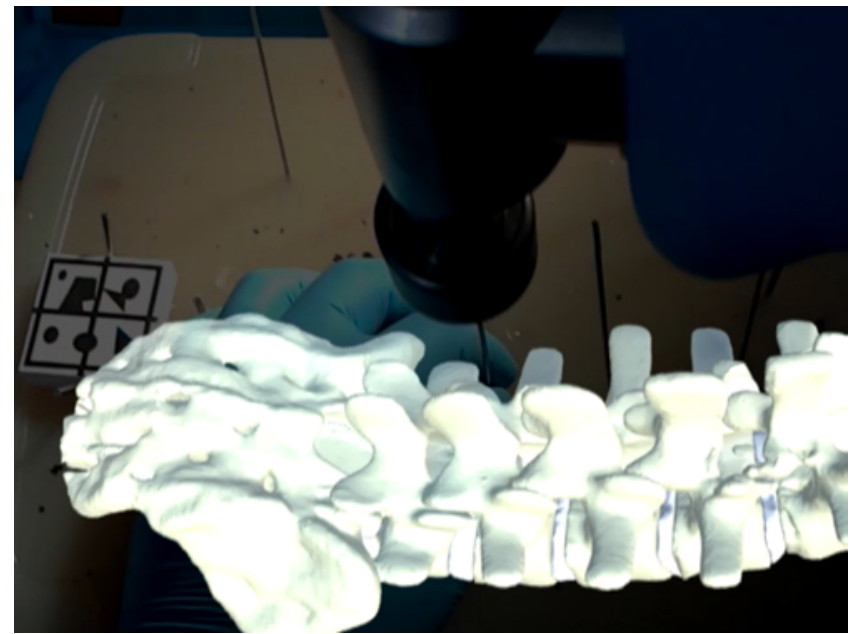

FIG. 3. A 300-mm-long K-wire with a 2.5-mm diameter was placed into the pedicle at a $45-\mathrm{mm}$ depth (HoloLens view). Figure is available in color online only.

coxon rank-sum test. A p value of 0.05 was set to indicate statistical significance. All statistical analyses were performed using Stata software (Stata 14.0, StataCorp LLC).

\section{Results}

Among all screw placements, 75 (94\%) of 80 AR-guided placements and 40 (100\%) of 40 radiograph-guided placements were acceptable (grade I or II; $p=0.106$; Table 1). Radiograph-guided placements had a larger proportion of grade I outcomes than the AR-guided method ( $\mathrm{p}$ $<0.0001$; Table 1). The accuracy of the two AR alignment methods ( $\mathrm{p}=0.526$; Table 1 ) was not significantly different, and neither was it different between the AR and radiograph groups ( $\mathrm{p}<0.0001$; Table 2$)$.

AR-guided placements required less time than radiograph-guided placements (mean \pm standard deviation, $131.76 \pm 24.57$ vs $181.43 \pm 15.82$ seconds, respectively, $\mathrm{p}$

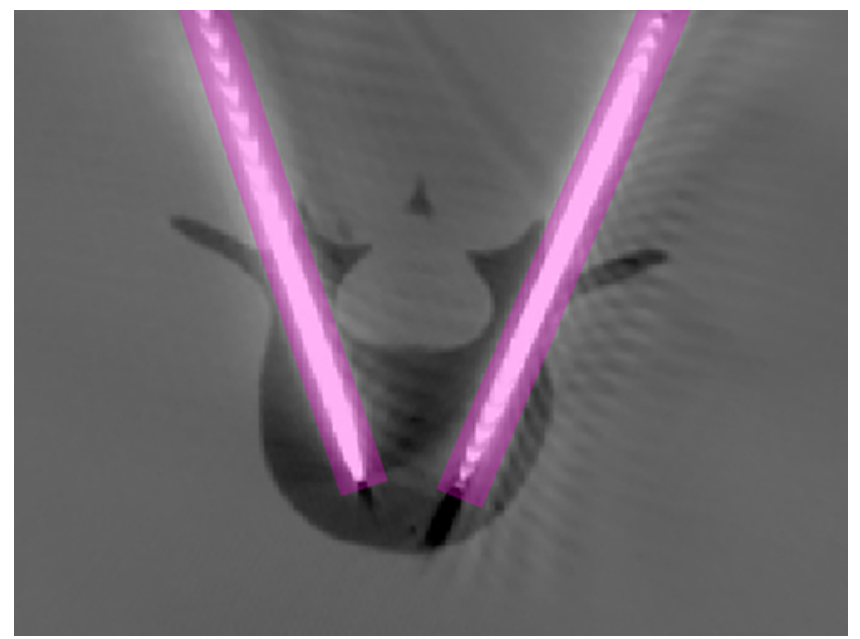

FIG. 4. For each K-wire, the insertion path was expanded to a 6.5-mm diameter. Figure is available in color online only. 
TABLE 1. Accuracy of AR-guided and radiograph-guided placements

\begin{tabular}{cccc}
\hline & \multicolumn{2}{c}{ AR-Guided Method } & \\
\cline { 2 - 3 } Variable & $\begin{array}{c}\text { Automatic } \\
\text { Alignment }\end{array}$ & $\begin{array}{c}\text { Manual } \\
\text { Alignment }\end{array}$ & $\begin{array}{c}\text { Radiograph-Guided } \\
\text { Method }\end{array}$ \\
\hline Accuracy & & & \\
\hline Grade I & 22 & 17 & 35 \\
\hline Grade II & 16 & 20 & 5 \\
\hline Grade III & 2 & 3 & 0 \\
\hline Total no. & 40 & 40 & 40 \\
\hline
\end{tabular}

Values are expressed as the number of pedicle screw placements.

$<0.0001$; Table 3). Placements using the automatic-alignment method required less time than those using the manual-alignment method $(124.20 \pm 23.80$ vs $139.33 \pm 23.21$ seconds, respectively, $\mathrm{p}=0.0081$; Table 4).

\section{Discussion}

The image-based navigation system has been developed and applied in spine surgery to improve the learning curve and surgical accuracy involved in the MIS procedure. ${ }^{12,18}$ Under the guidance of this system, multiplanar imaging reconstruction of the anatomical structures can be displayed onscreen and instruments can be tracked in real time. A typical surgical navigation system (StealthStation, Medtronic Sofamor Danek) combines intricate surgical hardware including an O-arm (Medtronic Sofamor Danek) allowing intraoperative CT, an infrared camera tracking both the patient and the instruments, and a computing station calculating and displaying the position of instruments. The parts, especially the $\mathrm{O}$-arm, are too large to fit in a crowded operation room. As with any other technique, there is also a learning curve in gaining proficiency with the navigation system. Surgeons must reconstruct the anatomical structures mentally through three onscreen images, based on a CT image, in projections different from the transverse, coronal, or sagittal planes and use special instruments with fixed infrared reflective fiducial spheres to track instruments during a procedure. Inappropriate grasping of instruments or blocking of the infrared ray path can lead to an inability to track.

AR technology can enhance visual perception through

TABLE 2. Accuracy of pedicle placements by two surgeons

\begin{tabular}{crrrrr}
\hline & \multicolumn{2}{c}{ Surgeon 1 } & & \multicolumn{2}{c}{ Surgeon 2 } \\
\cline { 2 - 3 } \cline { 5 - 6 } Variable & AR & Radiograph & & AR & Radiograph \\
\hline Accuracy & & & & & \\
\hline Grade I & 20 & 17 & & 19 & 18 \\
\hline Grade II & 17 & 3 & & 19 & 2 \\
\hline Grade III & 3 & 0 & & 2 & 0 \\
\hline Total no. & 40 & 20 & & 40 & 20 \\
\hline
\end{tabular}

Values are expressed as the number of pedicle screw placements.
TABLE 3. Surgical duration of AR-guided and radiograph-guided operations

\begin{tabular}{|c|c|c|c|c|c|}
\hline \multirow[b]{2}{*}{ Variable } & \multirow{2}{*}{$\begin{array}{c}\text { No. of } \\
\text { Placements }\end{array}$} & \multicolumn{4}{|c|}{ Time (secs) } \\
\hline & & Mean & SE & SD & $95 \% \mathrm{Cl}$ \\
\hline AR-guided op & 80 & 131.76 & 2.75 & 24.57 & $126.30,137.23$ \\
\hline $\begin{array}{l}\text { Radiograph- } \\
\text { guided op }\end{array}$ & 40 & 181.43 & 2.50 & 15.82 & $176.37,186.49$ \\
\hline Total no. & 120 & 148.32 & 2.94 & 32.18 & $142.50,154.13$ \\
\hline
\end{tabular}

superimposed information generated by a computer. Recently, many researchers have applied AR technology in MIS procedures, and several experimental trials of medical AR systems have been reported. Bichlmeier and colleagues have proposed the combination of a navigation system and AR technology and have completed accurate pedicle screw placements on spine phantoms. ${ }^{3}$ They used an infrared reflective fiducial sphere similar to that of a traditional navigation system to track the patient and instruments. The AR aspect was displayed in a special headmounted display. They used a traditional infrared navigation system to track and the AR exclusively to display.

Similar to our study, Agten et al. used the Microsoft HoloLens to perform AR-guided lumbar facet joint injections on a phantom. ${ }^{1}$ They had only two vertebrae embedded in a phantom, and alignment of the hologram was performed by a manual method matching three virtual markers to three adhesive tape markers fixed on the phantom. Each surgeon performed the same operation on the same phantom 20 times. Their study provided clear evidence to support the use of AR-guided lumbar facet joint injections. However, their study also used a larger needle than is typically used in their clinic, and they did not measure radiation, both of which they deemed as study flaws. Moreover, the phantom they used was too small, and we believe it is not difficult to achieve high puncture accuracy without any guidance. Their study focused only on the target location; however, the puncture path is also important. Additionally, there was no tracking system to locate the patient (the phantom) in their study. The HoloLens can scan the environment around the user and locate itself in the room, which is called "spatial mapping." However, the accuracy of this self-location is not satisfactory. When the wearer turns his or her head around and turns back to the initial position, the previously projected hologram is likely to drift away, and one or more realignments are needed.

Our study demonstrated that percutaneous lumbar pedicle screw placement was feasible using an AR guiding system in bone-agar experimental settings. We also demonstrated that automatic alignment of the hologram was more efficient than manual alignment and that the accuracy of the two alignment methods was not significantly different. Moreover, with AR guidance, the surgeon can focus on the surgical field and not on the navigation screen.

Our study had some limitations. The AR guiding system was not studied in a clinical setting with real-life patients. Moreover, the processes that must be performed after intraoperative CT were complicated, and obtaining 
TABLE 4. Duration of two alignment methods of the AR-guided operation

\begin{tabular}{cccccc}
\hline & \multirow{2}{*}{ No. of } & \multicolumn{4}{c}{ Time (secs) } \\
\cline { 3 - 6 } Variable & Placements & Mean & SE & SD & $95 \% \mathrm{Cl}$ \\
\hline Alignment & & & & & \\
\hline Automatic & 40 & 124.2 & 3.76 & 23.80 & $116.59,131.81$ \\
\hline Manual & 40 & 139.33 & 3.67 & 23.21 & $131.90,146.75$ \\
\hline Total no. & 80 & 131.76 & 2.75 & 24.57 & $126.30,137.23$ \\
\hline
\end{tabular}

segmented CT images and building a holographic application to deploy to the HoloLens can take 10-40 minutes to reconstruct. Additionally, 3D reconstruction software may not be freeware, and some applications can only export to STL files, which then must be converted to FBX files (e.g., using Unity3D, Unity Technologies) to transfer into the HoloLens software. Also, because the automatic alignment relies on image identification or gray template matching, the change in brightness or contrast of the image can cause identification failure. The brightness or contrast was considerable enough that moving persons in the operating room or moving hands around the image marker could lead to identification failure. Even after a successful identification, the field depth of the displayed hologram can be different from real life, and thus depth perception may be an issue. The user could align the instrument's direction or orientation to the right puncture path but would not know how far to advance to the target or could pass it. Thus, a depth limitation device was needed in the K-wire placement operation.

The accuracy of the AR guiding system was susceptible. Deviation of each process during the procedure can result in an error cascade. During the segmentation process after intraoperative $\mathrm{CT}$, reconstructed 3D models of the cubic marker were adjusted to coincide with a preprogrammed cubic anchor of the AR hologram. CT artifacts of metal fixators can decrease the smoothness of 3D models and further lower the coincidence rate. Micromovement of cubic markers fixed on phantoms can happen during the Kwire placement operation, which can lead to an imprecise anchor point of the AR hologram. Finally, accuracy is also determined by the HoloLens itself, whose accuracy would be affected even by varied brightness, contrast, or distance to the cubic marker. In our study, environmental factors of the operating room were controlled, and all operations were performed as gently as possible to avoid micromovement of the cubic markers. This rigorously controlled environment is obviously impractical in a clinical situation.

\section{Conclusions}

In bone-agar experimental settings, lumbar pedicle screw placements using the AR-guided percutaneous method were acceptable and more efficient than radiograph-guided placements. In the AR-guided placements, the automatic-alignment method was as accurate as the manual-alignment method but more efficient. Because of the limitations of this study, further investigation in clini- cal settings is warranted to clarify the safety and efficacy of this procedure. Importantly, the AR guiding system cannot be recommended until significant improvement of the alignment and tracking methods is accomplished.

\section{Acknowledgments}

This research was supported by the clinical research project of the Second Affiliated Xinqiao Hospital of Army Medical University (grant no. 2018JSLC0014).

\section{References}

1. Agten CA, Dennler C, Rosskopf AB, Jaberg L, Pfirrmann CWA, Farshad M: Augmented reality-guided lumbar facet joint injections. Invest Radiol 53:495-498, 2018

2. Azuma RT: A survey of augmented reality. Presence (Camb Mass) 6:355-385, 1997

3. Bichlmeier C, Heining SM, Feuerstein M, Navab N: The virtual mirror: a new interaction paradigm for augmented reality environments. IEEE Trans Med Imaging 28:1498-1510, 2009

4. Chatzopoulos D, Bermejo C, Huang Z, Hui P: Mobile augmented reality survey: from where we are to where we go. IEEE Access 5:6917-6950, 2017

5. Foley KT, Holly LT, Schwender JD: Minimally invasive lumbar fusion. Spine (Phila Pa 1976) 28 (15 Suppl):S26-S35, 2003

6. Foley KT, Lefkowitz MA: Advances in minimally invasive spine surgery. Clin Neurosurg 49:499-517, 2002

7. Fritz J, U-Thainual P, Ungi T, Flammang AJ, Cho NB, Fichtinger G, et al: Augmented reality visualization with image overlay for MRI-guided intervention: accuracy for lumbar spinal procedures with a 1.5-T MRI system. AJR Am J Roentgenol 198:W266-W273, 2012

8. Fritz J, U-Thainual P, Ungi T, Flammang AJ, Fichtinger G, Iordachita II, et al: Augmented reality visualization with use of image overlay technology for MR imaging-guided interventions: assessment of performance in cadaveric shoulder and hip arthrography at 1.5 T. Radiology 265:254-259, 2012

9. George DC, Krag MH, Johnson CC, Van Hal ME, Haugh LD, Grobler LJ: Hole preparation techniques for transpedicle screws. Effect on pull-out strength from human cadaveric vertebrae. Spine (Phila Pa 1976) 16:181-184, 1991

10. Hohl JB, Holt DC, Brodke DS: Minimally invasive spine surgery complications with implant placement and fixation, in Phillips F, Lieberman I, Polly D (eds): Minimally Invasive Spine Surgery. Berlin: Springer, 2014, pp 431-444

11. Huang Z, Hui P, Peylo C, Chatzopoulos D: Mobile augmented reality survey: a bottom-up approach. arXiv:1309.4413, 2013

12. Innocenzi G, Bistazzoni S, D'Ercole M, Cardarelli G, Ricciardi F: Does navigation improve pedicle screw placement accuracy? Comparison between navigated and non-navigated percutaneous and open fixations. Acta Neurochir Suppl 124:289-295, 2017

13. McAfee PC, Phillips FM, Andersson G, Buvenenadran A, Kim CW, Lauryssen C, et al: Minimally invasive spine surgery. Spine (Phila Pa 1976) 35 (26 Suppl):S271-S273, 2010

14. Ofiram E, Polly DW, Gilbert TJ Jr, Choma TJ: Is it safer to place pedicle screws in the lower thoracic spine than in the upper lumbar spine? Spine (Phila Pa 1976) 32:49-54, 2007

15. Park Y, Ha JW: Comparison of one-level posterior lumbar interbody fusion performed with a minimally invasive approach or a traditional open approach. Spine (Phila Pa 1976) 32:537-543, 2007

16. Perez-Cruet MJ, Fessler RG, Perin NI: Review: complications of minimally invasive spinal surgery. Neurosurgery $\mathbf{5 1}$ (5 Suppl):S26-S36, 2002 
17. Rasouli MR, Rahimi-Movaghar V, Shokraneh F, MoradiLakeh M, Chou R: Minimally invasive discectomy versus microdiscectomy/open discectomy for symptomatic lumbar disc herniation. Cochrane Database Syst Rev (9):CD010328, 2014

18. Tajsic T, Patel K, Farmer R, Mannion RJ, Trivedi RA: Spinal navigation for minimally invasive thoracic and lumbosacral spine fixation: implications for radiation exposure, operative time, and accuracy of pedicle screw placement. Eur Spine J 27:1918-1924, 2018

19. Tormenti MJ, Maserati MB, Bonfield CM, Okonkwo DO, Kanter AS: Complications and radiographic correction in adult scoliosis following combined transpsoas extreme lateral interbody fusion and posterior pedicle screw instrumentation. Neurosurg Focus 28(3):E7, 2010

20. Virk SS, Yu E: The top 50 articles on minimally invasive spine surgery. Spine (Phila Pa 1976) 42:513-519, 2017

\section{Disclosures}

The authors report no conflict of interest concerning the materials or methods used in this study or the findings specified in this paper.

\section{Author Contributions}

Conception and design: Zhou, Liu, C Li. Acquisition of data: Liu. Analysis and interpretation of data: Liu, Wang. Drafting the article: Liu. Critically revising the article: Zhou, Liu, Wu, Tang, C Li. Reviewed submitted version of manuscript: Liu, C Li. Statistical analysis: Liu, C Li. Administrative/technical/material support: Liu, C Li. Study supervision: Liu, H Li, C Li.

\section{Correspondence}

Yue Zhou: The Second Affiliated Xinqiao Hospital of Army Medical University, Chongqing, China. happyzhou@vip.163.com. 